\title{
Nutritional influences on human neurocognitive functioning
}

\author{
Michael A. Smith ${ }^{1 *}$ and Andrew B. Scholey ${ }^{2}$ \\ ${ }^{1}$ Department of Psychology, Faculty of Health and Life Sciences, Northumbria University, Newcastle upon Tyne, UK \\ ${ }^{2}$ Centre for Human Psychopharmacology, Swinburne University of Technology, Melbourne, VIC, Australia \\ ${ }^{*}$ Correspondence: michael4.smith@northumbria.ac.uk
}

Edited and reviewed by:

Hauke R. Heekeren, Freie Universität Berlin, Germany

Keywords: nutrition, diet, brain, neurocognitive functioning, neuroimaging

The notion that good nutrition is essential for adequate growth and sound physical wellbeing is very well established. Further, in recent years, there has been an overwhelming increase in research dedicated to better understanding how nutritional factors influence cognition and behavior (Riby et al., 2012). An aim of this Research Topic was to bring together Review, Opinion and Original Research articles reflecting the current science in this discipline. These include the effects of a range of foods and nutritional substrates on acute and chronic human neurocognitive functioning. The 13 accepted papers which form this Research Topic cover a diverse range of topics relating nutritional factors to neurocognitive functioning and performance. The articles demonstrate that neurocognitive performance is influenced by nutritional factors ranging from the dietary level (e.g., whole diet and meal composition) through to effects of macronutrients (such as glucose and omega-3 fatty acids) and micronutrients (vitamins, iron) on neurocognitive performance.

An objective of this research topic was to consider how various nutritional factors impact upon neurocognitive functioning at different stages of the lifespan. A number of the submissions focused on effects of nutrition in childhood, during which time nutrition plays an important role in growth and development, including via influences on constituents of the human central nervous system. A review by Nyaradi et al. (2013) considered the role of nutrition from a very broad perspective on neurocognitive development from the prenatal period through to childhood. This suggested that while observational studies have supported an important role for several individual nutrients (such as omega-3 fatty acids, B vitamins, iron) in the neurocognitive development of children, intervention studies aimed at supplementing intake of these individual nutrients have demonstrated inconclusive benefits. The authors of this review also highlighted the beneficial neurocognitive effects of breastfeeding and regular breakfast consumption as well as the impairing neurocognitive effects of childhood malnutrition. Kitsao-Wekulo et al. (2013) aimed to extend current understanding of this link between childhood malnutrition and poor cognitive outcomes, by investigating nutritional status as a mediator of the relationship between several socio-demographic variables and cognitive function in a sample of predominantly rural-dwelling Kenyan children. Nutritional status was found to mediate the relationship between socio-demographic factors and (i) language, (ii) motor function, and (iii) executive functioning in this study. With respect to specific micronutrient deficiencies that translate to adverse neurocognitive outcomes, Radlowski and Johnson (2013) reviewed the literature relating to the most common global nutrient deficiency, namely iron deficiency. They report that maternal anemia during the perinatal period increases the risk of delayed neurocognitive development. A further nutrient for which intake is typically below recommended levels in Western individuals is the omega-3 docosahexaenoic acid (DHA). Low dietary levels of this essential fatty acid are potentially problematic given (i) the involvement of this nutrient in mediating several critical brain functions, and (ii) DHA is derived from the diet alone. Similarly to the review of Nyaradi et al. (2013), Heaton et al. (2013) review concludes that dietary and plasma DHA levels in infancy appear to be associated with enhanced cognitive development, but that RCTs investigating infant DHA supplementation have been inconclusive with respect to beneficial effects on cognitive development. However, these authors note substantial methodological issues with RCTs of infant DHA supplementation studies, which could in part explain the equivocal findings (see also Meldrum et al., 2011). In a further review by Whiteley et al. (2013), it was argued that several dietary interventions have been effective in attenuating the neurocognitive and other adverse psychological outcomes in developmental disorders. The authors focused specifically on an intervention involving dietary elimination of gluten (the major protein in wheat, barley and rye) and casein (found in mammalian dairy products), and reported that this gluten and casein free dietary intervention was effective in enhancing such functions as language, attention and motor control in individuals with autism spectrum disorders.

Caroline Edmonds has conducted several studies investigating the influence of hydration status on cognitive functioning, with previous studies observing that access to water improves cognitive performance in children (Edmonds and Jeffes, 2009). In the paper included in this Research Topic, Edmonds et al. (2013) observed that beneficial effects of water consumption may be limited to individuals with relatively higher levels of subjective thirst, with thirsty individuals who were not provided with water exhibiting slower simple reaction times compared with (i) those who were administered water and (ii) those who were not administered water but reported lower levels of subjective thirst. In a further empirical study, Gibson et al. (2013) found that younger women with a higher dietary intake of saturated fat showed deficits in learning and memory.

Three papers accepted into our Research Topic considered the role of breakfast, which has been argued by many nutritionists 
to be the "most important meal of the day," in neurocognitive performance. A review by Adolphus et al. (2013) reported that (i) the quality and frequency of the habitual breakfast meal and (ii) engagement with school breakfast programmes in children and adolescents influences academic attainment. In addition Defeyter and Russo (2013) investigated the acute effect of breakfast consumption (compared to fasting) in adolescent nonhabitual breakfast consumers, and observed that breakfast consumption enhanced verbal memory (under conditions of greater cognitive load) and backwards counting performance. However, no effects were observed in a range of other cognitive domains. Conversely, Zilberter and Zilberter (2013) highlight the equivocal findings of previous studies investigating the relationship between breakfast consumption and neurocognitive performance. These authors report that several different breakfast effects which have been investigated previously (e.g., glycemic load of the breakfast meal, nutritional composition, breakfast vs. no breakfast) have yielded positive, negative, and null effects on neurocognitive performance across a range of different populations under investigation. Thus it appears that more studies are needed to ascertain the specific benefits of breakfast on neurocognitive performance.

Finally, in recent years neuroimaging studies have made a substantial contribution to our understanding of the neurocognitive mechanisms underpinning nutritional influences on human cognitive performance. Three papers within this Research Topic specifically discuss the role of neuroimaging in investigating the link between nutrition and cognitive functioning. With respect to carbohydrate intake and neurocognitive performance, it is well established that glucose ingestion enhances memory performance, but no such beneficial memory effect of glucose is typically observed for emotionally laden stimuli (Smith et al., 2011). Schopf et al. (2013) report that following glucose ingestion, the hypothalamus becomes inactive in response to emotional material, providing a mechanistic explanation for the previously observed behavioral observations. Further, Jackson and Kennedy (2013) discuss the ways in which near-infrared spectroscopy has proven useful in detecting changes in cerebral blood flow following ingestion of dietary constituents including caffeine, polyphenols and omega- 3 fatty acids. A paper which reviewed the literature relating to neuroimaging studies that have investigated the mechanisms underpinning the influence of early diet on cognitive and brain development by Isaacs (2013) provides a sound overview of the work which has been conducted on this topic.

In summary, it is clear that nutritional status, diet and the ingestion of a range of nutrients impacts upon neurocognitive development, function, and performance. The papers within this Research Topic consider a range of these effects. However, equivocal findings have emerged from many studies which have investigated the relationship between nutrition and cognition. Neuroimaging studies are informative with respect to the precise mechanisms which mediate these effects, and future studies in this area will contribute greatly to our understanding of the relationship between nutrition, diet and human neurocognitive functioning.

\section{REFERENCES}

Adolphus, K., Lawton, C. L., and Dye, L. (2013). The effects of breakfast on behavior and academic performance in children and adolescents. Front. Hum. Neurosci. 7:425. doi: 10.3389/fnhum.2013. 00425

Defeyter, M. A., and Russo, R. (2013). The effect of breakfast cereal consumption on adolescents' cognitive performance and mood. Front. Hum. Neurosci. 7:789. doi: 10.3389/fnhum.2013.00789

Edmonds, C. J., Crombie, R., and Gardner, M. R. (2013). Subjective thirst moderates changes in speed of responding associated with water consumption. Front. Hum. Neurosci. 7:363. doi: 10.3389/fnhum.2013.00363

Edmonds, C. J., and Jeffes, B. (2009). Does having a drink help you think? 6-7-Year-old children show improvements in cognitive performance from baseline to test after having a drink of water. Appetite 53, 469-472. doi: 10.1016/j.appet.2009.10.002

Gibson, E. L., Barr, S., and Jeanes, Y. M. (2013). Habitual fat intake predicts memory function in younger women. Front. Hum. Neurosci. 7:838. doi: 10.3389/fnhum.2013.00838

Heaton, A. E., Meldrum, S. J., Foster, J. K., Prescott, S. L., and Simmer, K. (2013). Does docosahexaenoic acid supplementation in term infants enhance neurocognitive functioning in infancy? Front. Hum. Neurosci. 7:774. doi: 10.3389/fnhum.2013.00774

Isaacs, E. B. (2013). Neuroimaging, a new tool for investigating the effects of early diet on cognitive and brain development. Front. Hum. Neurosci. 7:445. doi: 10.3389/fnhum.2013.00445

Jackson, P. A., and Kennedy, D. O. (2013). The application of near infrared spectroscopy in nutritional intervention studies. Front. Hum. Neurosci. 7:473. doi: 10.3389/fnhum.2013.00473

Kitsao-Wekulo, P., Holding, P., Taylor, H. G., Abubakar, A., Kvalsvig, J., and Connolly, K. (2013). Nutrition as an important mediator of the impact of background variables on outcome in middle childhood. Front. Hum. Neurosci. 7:713. doi: $10.3389 /$ fnhum.2013.00713

Meldrum, S. J., Smith, M. A., Prescott, S. L., Hird, K., and Simmer, K. (2011). Achieving definitive results in long-chain polyunsaturated fatty acid supplementation trials of term infants: factors for consideration. Nutr. Rev. 69, 205-214. doi: 10.1111/j.1753-4887.2011.00381.x

Nyaradi, A., Li, J., Hickling, S., Foster, J., and Oddy, W. H. (2013). The role of nutrition in children's neurocognitive development, from pregnancy through childhood. Front. Hum. Neurosci. 7:97. doi: 10.3389/fnhum.2013.00097

Radlowski, E. C., and Johnson, R. W. (2013). Perinatal iron deficiency and neurocognitive development. Front. Hum. Neurosci. 7:585. doi: 10.3389/fnhum.2013.00585

Riby, L. M., Smith, M. A., and Foster, J. K., (eds.). (2012). Nutrition and Mental Performance: A Lifespan Perspective. London: Palgrave MacMillan.

Schopf, V., Fischmeister, F. P., Windischberger, C., Gerstl, F., Wolzt, M., Karlsson, K. A. E., et al. (2013). Effects of individual glucose levels on the neuronal correlates of emotions. Front. Hum. Neurosci. 7:212. doi: 10.3389/fnhum.2013.00212

Smith, M. A., Riby, L. M., Eekelen, J. A., and Foster, J. K. (2011). Glucose enhancement of human memory: a comprehensive research review of the glucose memory facilitation effect. Neurosci. Biobehav. Rev. 35, 770-783. doi: 10.1016/j.neubiorev.2010.09.008

Whiteley, P., Shattock, P., Knivsberg, A. M., Seim, A., Reichelt, K. L., Todd, L., et al. (2013). Gluten- and casein-free dietary intervention for autism spectrum conditions. Front. Hum. Neurosci. 6:344. doi: 10.3389/fnhum.2012.00344

Zilberter, T., and Zilberter, E. Y. (2013). Breakfast and cognition: sixteen effects in nine populations, no single recipe. Front. Hum. Neurosci. 7:631. doi: 10.3389/fnhum.2013.00631

Conflict of Interest Statement: Andrew Scholey has received research funding and consultancy from the health supplement industry. The authors declare that the manuscript was prepared in the absence of any commercial or financial relationships that could be construed as a potential conflict of interest.

Received: 02 April 2014; accepted: 09 May 2014; published online: 27 May 2014. Citation: Smith MA and Scholey AB (2014) Nutritional influences on human neurocognitive functioning. Front. Hum. Neurosci. 8:358. doi: 10.3389/fnhum.2014.00358 This article was submitted to the journal Frontiers in Human Neuroscience.

Copyright (๑) 2014 Smith and Scholey. This is an open-access article distributed under the terms of the Creative Commons Attribution License (CC BY). The use, distribution or reproduction in other forums is permitted, provided the original author $(s)$ or licensor are credited and that the original publication in this journal is cited, in accordance with accepted academic practice. No use, distribution or reproduction is permitted which does not comply with these terms. 